\title{
ACCESO Y PERMANENCIA EN LA EDUCACIÓN SUPERIOR: ESTRATEGIAS PARA SU MEJORAMIENTO. EL CASO DE LA UNS
}

\author{
Silvia Morresi*
}

enviado: marzo 2013-aceptado: mayo 2014

\section{INTRODUCCIÓN}

A partir del reconocimiento de la relación entre niveles de educación e ingreso y de que los procesos de reestructuración y la vigencia de nuevos paradigmas empresariales han generado un cambio en el patrón de la demanda de calificaciones de los trabajadores, alcanza relevancia la vinculación entre el sistema educativo y el productivo y entre la educación y el trabajo. El reconocimiento de esta vinculación se evidencia tanto a nivel de las autoridades como de la población en general. La problemática de la educación ocupa un lugar destacado en la agenda de la mayoría de los países, siendo la expresión más abarcativa en el caso de los países iberoamericanos el proyecto Metas 2021, que en general propugna ampliar el acceso a una educación de calidad en todos sus niveles. Muestra de la importancia otorgada por la población en general a la educación ya sea como mecanismo para mejorar la calidad de vida o como el medio para contar con nuevas "credenciales" o como el camino para incorporar un mayor y diversificado caudal de conocimientos para lograr una posición más competitiva en el mercado laboral es el incremento sostenido en la matrícula. En cuanto a la educación superior, si bien en general el ingreso a las instituciones universitarias es irrestricto y el servicio educativo es gratuito, existe evidencia que no sustenta la existencia de igualdad de oportunidades entre educandos de distintos sectores sociales de manera que aquellos con mayores desventajas iniciales puedan acceder a condiciones de aprendizaje equivalentes. $\mathrm{La}$ finalidad de esta ponencia será indagar acerca de las estrategias implementadas por las

\footnotetext{
* Departamento de Economía - Universidad Nacional del Sur; Instituto de Investigaciones Económicas y Sociales del Sur (IIESS) - UNS, CONICET. smorresi@uns.edu.ar
} 
autoridades competentes para propiciar el acceso y permanencia de los estudiantes en la educación de tercer nivel. A tal fin en primer término se caracterizara brevemente la evolución y la situación actual del nivel de educación superior argentino en general y en particular se presentaran consideraciones referentes a la Universidad Nacional del Sur. Luego se reseñaran las causas que motivan a los estudiantes a abandonar sus estudios y se seleccionaran un conjunto de variables que permitan definir el perfil de los alumnos de la UNS que han logrado graduarse para contrastarlo con el de aquellos que han desertado. En la sección siguiente se analizan los alcances de los programas implementados a nivel nacional y a nivel de la UNS para mejorar el acceso y desempeño de los alumnos del sistema.

\section{NIVEL DE EDUCACIÓN SUPERIOR. EVOLUCIÓN Y SITUACIÓN ACTUAL. ALGUNOS RASGOS}

El nivel de educación superior en la Argentina está compuesto por Universidades, Institutos Universitarios e Institutos de Educación Superior. Las características distintivas de la evolución de la educación superior en las últimas décadas fueron la expansión y la diferenciación. Estas transformaciones provocaron entre otros efectos la conformación de un sistema binario con importante presencia de instituciones privadas, la expansión territorial de las instituciones más allá de las ciudades capitales y grandes centros urbanos, -ya sea mediante la instalación de nuevas instituciones o la apertura de sedes y programas, cuyo campus principal se halla ubicado en otra ciudad - y el aumento y diversificación de la oferta educativa a partir de la incorporación de nuevas carreras de grado y posgrado. Entre los aspectos relevantes de este proceso pueden señalarse: el diseño y ejecución de sistemas de control de la calidad, la implementación en algunas instituciones de mecanismos de admisión para los alumnos ingresantes, la asignación de los recursos en función de variables de gestión, la pertinencia y la articulación de las instituciones de educación superior con el contexto social y económico de las áreas y regiones en las que se encuentran insertas. Desde esta perspectiva, la integración de las casas de estudio en los entramados de actores para el desarrollo asume una importancia central en razón de su potencial de creación y transferencia de conocimiento, un activo clave para la innovación y el desempeño socioeconómico de las regiones y los territorios (Rama, 2004).

Estos cambios, junto al crecimiento de la población de estudiantes del nivel secundario $^{1}$; la necesidad de creciente capacitación frente a las transformaciones

1 Entre 1993 y 2010 la tasa neta de matriculación secundaria se incrementó en 30.6 puntos porcentuales (SITEAL), alcanzando una cobertura superior al 70\%. (Rama, C. 2009) 
introducidas en los procesos productivos que impactan sobre el modo de utilización del trabajo en relación al tipo de calificaciones, saberes, destrezas, habilidades, información, actitudes, y atributos de personalidad que se vuelcan en el proceso productivo, y la evidencia de tasas más elevadas de ingresos y menores tasas de desocupación de los egresados de nivel superior en relación con el resto de la población ocupada ${ }^{2}$, alentaron por un lado el aumento en la demanda por educación superior y por otro delinearon un nuevo perfil de la matrícula universitaria.

En este sentido y atendiendo solo al sistema universitario puede observarse que si bien entre las instituciones encargadas de proveer servicios educativos predominan las de carácter privado, absorben solo un quinto de la matrícula (Cuadro 1).

La evolución del número de alumnos en el sistema de educación superior muestra un fuerte crecimiento durante los últimos 30 años y continúa expandiéndose a tasas que fluctúan en torno al 2,5\% anual. Esta evolución presenta algunas particularidades. Por un lado en las instituciones de gestión privada, durante el periodo 2000-2010, la tasa de crecimiento de su alumnado alcanza guarismos (5.8\%), que supera en 4 puntos porcentuales a la tasa correspondiente a las instituciones de gestión estatal. Por otro lado se observa en este último sector, en los años recientes, la conformación de dos grupos de universidades con dinámicas distintas: uno compuesto mayoritariamente por universidades tradicionales o universidades de más antigua creación, que enfrentaron cierta disminución de su matrícula y otro donde predominan las universidades más nuevas, con un aumento sostenido del número de estudiantes (Proyecto Institucional de la UNLu 2009-2012).

En este punto resulta de interés analizar la evolución de las tasas de participación de los inscriptos en programas de educación superior en relación a la cohorte en edad de cursar estudios superiores. Esta tasa se ha duplicado durante los últimos años, alcanzando valores que permiten situar al sistema argentino, de acuerdo a la clasificación de Rama (2009), en una etapa de masificación ${ }^{3}$. Esta

2 Diversos trabajos empíricos en nuestro país entre los que pueden citarse Di Paola et al. (1997), Perlbach de Maradona et al., (1997), Petrecolla, D. et al. (1997), muestran que la inversión en capital humano se constituye en una variable explicativa de los diferenciales de ingresos de la población, también en informes de la OCDE se muestra esta relación (Ministerio de educación, 2011)

3 Rama (2009) a partir del trabajo Martín Trow (1974) en el que sostiene que el desarrollo de la educación superior puede organizarse en tres etapas: elite, masas y universal - cuyos parámetros establece en términos de la tasa bruta de matriculación en la universidad- elabora un esquema más diferenciado buscando determinar mayor cantidad de peldaños que permitan distinguir más claramente los sistemas de educación superior en los actuales contextos, sugiriendo un acceso de elites hasta el 15\%, un acceso de minorías del 15 al 30\%, de masas hasta el 50\%, universal hasta el $85 \%$ y un acceso absoluto quedaría indicado con porcentaje superior a este último. 
etapa ha sido acompañada por una creciente feminización de la inscripción en el ciclo universitario (Cuadro 1).

Las posibilidades de participar en la educación superior está condicionada por el origen socio-económico de los alumnos, encontrándose que el porcentaje de personas de 20 a 24 años incorporadas al sistema, que provienen de los hogares de menores ingresos, está sensiblemente por debajo de la tasa de escolarización correspondiente a la totalidad de ese estrato etario (Cuadro 1).

Cuadro 1. Sistema de educación superior. Algunas características

\begin{tabular}{|c|c|c|}
\hline Instituciones universitarias según sector de gestión & $\begin{array}{l}\text { Publicas } \\
\text { Privadas }\end{array}$ & $\begin{array}{l}55 \\
57\end{array}$ \\
\hline Estudiantes de carreras de grado y pregado & $\begin{array}{l}1975 \\
2000 \\
2010\end{array}$ & $\begin{array}{c}5792736 \\
1.339 .740 \\
1.718 .507\end{array}$ \\
\hline $\begin{array}{l}\text { Tasa bruta de matriculación universitaria } \\
\text { (rango OCDE) }(\%)\end{array}$ & & 52,1 \\
\hline $\begin{array}{l}\text { Participación de la matricula de instituciones de gestión } \\
\text { privada en la matricula total }(\%)\end{array}$ & & 20,5 \\
\hline Género & $\begin{array}{l}\text { Masculino } \\
\text { Femenino }\end{array}$ & $\begin{array}{l}43,9 \\
56,1\end{array}$ \\
\hline $\begin{array}{l}\text { Asistencia a instituciones de educación superior de } \\
\text { personas del primer quintil de ingreso (\%) }\end{array}$ & & 22,9 \\
\hline
\end{tabular}

Fuente: elaboración propia en base a Cinda (2007), SPU (2010)

La Universidad Nacional del Sur como integrante del sistema de universidades nacionales, participa en el proceso de transformación de la educación superior ${ }^{4}$. A partir de mediados de la década del 90 inicia un importante proceso de diversificación y ampliación de su oferta educativa, incorporando tanto carreras tradicionales - Lic. en Ciencias de la Administración, Farmacia, Abogacía,

4 Cfr Donnini, N., Morresi, S. "El de las ciencias sociales en el diseño curricular de la universidad" Revista del $V$ Encuentro de Cátedras de Ciencias Sociales y Humanisticas para las Ciencias Económicas, Rosario 1998 
Medicina, Lic. en Enfermería entre otras- como diversas tecnicaturas. En el marco de este proceso, a fines de la misma década, comenzó a dictar en varias localidades de su región de influencia distintas tecnicaturas con duración de tres años, con posibilidades de articular luego con carreras de la propia Universidad. Estas tecnicaturas se enmarcan en el Programa de estudios universitarios en la zona $(\mathrm{PEUZO})^{5}$. La oferta académica está actualmente constituida por 64 carreras de grado y numerosas carreras de postgrado.

Junto al cambio cuantitativo, se producen cambios cualitativos entre los que se inscribe el fenómeno de la deserción. De los alumnos que ingresan anualmente a las universidades argentinas, solo una cuarta parte logra graduarse, en tanto que más del 75\% de ellos han abandonado sus estudios o bien se han cambiado de institución al finalizar el primer año de estudios (Ministerio de Educación, Ciencia y Tecnología, 2008). Estudios realizados en la Universidad Nacional del Sur para las cohortes 1986 y 1991 arrojan tasas brutas de abandono del 72,1 y 68,7 respectivamente. Esta estimación se realizó a diez años del inicio para la primera cohorte y a ocho años del comienzo de la segunda (Guevara, Cerioni, Donnini, Morresi 1998, 2000).

\section{DESERCIÓN EN EL NIVEL SUPERIOR: CONSIDERACIONES ACERCA DE SUS CAUSAS}

Las problemáticas más significativas con las que los alumnos deben enfrentarse al iniciar estudios superiores y que influyen sobre la continuidad de los mismos pueden ser diferentes según en qué etapa del ciclo de vida esté la persona, según sus capacidades o necesidades económicas. En este sentido los motivos del abandono pueden agruparse en causas inherentes o propias del estudiante conformando su bagaje personal (historia de vida, patrimonio cultural, hábitos de

5 La implementación del Programa de Estudios Universitarios en la Zona de la UNS, se justifica en fundamentos de diversa naturaleza, de carácter cultural que apuntan a reducir la brecha cultural que puede apreciarse entre las ciudades de la región frente a aquéllas otras que cuentan con universidades; de carácter socio-demográfico intentando evitar el drenaje progresivo de población joven que sufren las localidades de la región. Se persigue lograr con la implantación de las carreras propias (tecnicaturas) que los universitarios una vez graduados encuentren alternativas laborales en su lugar de origen. Cfr: Guevara, E.O. de , Cerioni L., Donnini, N., Morresi, S., "La Educación Superior y las Perspectivas Laborales a la luz de Algunos Indicadores de Desempeño", V Congreso Asociación de Especialistas en Estudios del Trabajo, Buenos Aires, Agosto 2001. Guevara, E.O. de, Donnini, N. "Nuevas formas educativas universitarias y cambios en el mercado laboral regional”, III Congreso Latinoamericano de Sociología del Trabajo, Buenos Aires, Mayo de 2000. 
estudio, vocación, modalidades de estudio diferentes, entre otros), en cuestiones relacionadas con el medio familiar en el que se encuentra inmerso, que pueden actuar como facilitador $u$ obstaculizador en su meta académica (estructura familiar, nivel de ingresos, situación laboral, etc.). Pero también las causales anidan en el plano institucional: desfasajes estructurales en la enseñanza primaria y secundaria; deficiencias en los dispositivos institucionales -interuniversitarios- destinados a garantizar la retención, permanencia y egreso en tiempo promedio; y también la ausencia de políticas -desde los estamentos ministeriales competentes- dirigidas a enfrentar el problema aulas adentro, y revertirlo (Ministerio de Educación, Ciencia y Tecnología, 2008).

La participación relativa de las distintas causas del abandono, no es uniforme ni en el tiempo, ni en las distintas comunidades educativas. A partir de la respuesta de los desertores de diversas cohortes de alumnos de la Universidad Nacional del Sur, acerca de las razones que motivaron su alejamiento de la institución puede decirse que las causas más frecuentes son pérdida de interés por la carrera y los motivos de orden económico. Con respecto a las otras dos causas más citadas, dificultades de estudio y deficiencias en la organización de la UNS, su relevancia difiere entre carreras y cohortes. De los alumnos que manifestaron como causa del abandono de sus estudios razones de índole económica, en promedio el 70\% de ellos cubría sus gastos con aportes familiares y trabajo personal, un grupo pequeño recibía financiamiento de otras fuentes tales como becas y el resto se autofinanciaba desarrollando en general actividades que no guardaban relación con su carrera (Cerioni et al., 2006; Guevara et al., 1998).

El análisis de las consideraciones realizadas por aquellos estudiantes que aducen pérdida de interés por la carrera como causa de deserción evidencia una cierta indefinición en términos vocacionales (Cerioni et al., 2006; Guevara et al., 1998).

La mayoría de los desertores que manifiestan que la dificultad en los estudios es una de las causas que influyó en su decisión de abandonar los claustros universitarios sostienen que la capacitación obtenida en el ciclo secundario resultó insuficiente debido, entre otras causas a que encontraron incompatibilidades entre el tipo de estudios que allí realizaron y el requerido en su carrera universitaria, a que el nivel de enseñanza en ese ciclo fue insuficiente, o a que no lograron formar en el ciclo secundario hábitos de estudios adecuados (Cerioni et al., 2006; Guevara, 1998).

Si bien el ingreso a las universidades argentinas de gestión pública es irrestricto y el servicio educativo es gratuito al comparar las características socioeconómicas del hogar de donde provienen los alumnos que abandonan sus 
estudios con el de los estudiantes que logran finalizar su carrera se encuentran marcadas diferencias, encontrándose que la mayor probabilidad de graduación se concentra en los jóvenes de origen socioeconómico alto (Fanelli et al, 2010). Un estudio realizado en la Universidad Nacional del Sur (UNS) en el año 1998 revela por un lado que el porcentaje de padres de familia ocupados como jefes, gerentes, directores o altos jefes, y dueños de empresa, pertenecientes a las clases sociales media alta y alta, representan una proporción mayor entre los padres de los egresados que de los desertores. Y por otro que los padres de los egresados tienen un nivel educacional más alto.

\section{ACCIONES ORIENTADAS A ATENDER LA PROBLEMÁTICA DEL ABANDONO DE LA EDUCACIÓN SUPERIOR}

Frente a esta realidad en la UNS se han implementado una serie de medidas tendientes a morigerar las problemáticas experimentadas por los alumnos en su tránsito por la universidad. Algunas de estas medidas están orientadas a mitigar cuestiones relacionadas con las dificultades de orden económico y otras a atender las derivadas de cuestiones académicas.

III.1. Estrategias destinadas a mejorar el rendimiento académico

Entre las estrategias destinadas a mejorar el desempeño de los alumnos pueden citarse los Proyectos de Mejoramiento de la Enseñanza, el Programa de Articulación Escuelas Medias Universidad y los Proyectos de Apoyo Estos proyectos fueron impulsados por la Secretaria de Políticas Universitarias (SPU).

En el marco del los Proyectos de Mejoramiento de la Enseñanza se desarrollaron el Proyecto de Mejoramiento de la Enseñanza en Ingeniería (PROMEI I y II) -con período de implementación 2005-2010-, el Proyecto de Mejoramiento de la Enseñanza en Agronomía (PROMAGRO) implementado durante 2007-2009 y el Proyecto de Mejoramiento de la Enseñanza en Farmacia y Bioquímica (PROMFYB) vigente durante 2008-2010. Estos proyectos consisten en apoyo financiero otorgado a las instituciones para destinarlo a la superación de las debilidades señaladas en el proceso de acreditación llevado adelante por la Comisión Nacional de Evaluación y Acreditación Universitaria (CONEAU). La evaluación de la tasa de retención o reinscripción de los alumnos en las carreras alcanzadas por estos programas muestra una tendencia creciente que permitiría augurar un mejoramiento en la tasa de graduación (Cuadro 2). 
Cuadro 2. UNS. Ingenierías. Algunos indicadores

\begin{tabular}{lccc}
\hline & 2000 & 2005 & 2010 \\
\cline { 2 - 4 } $\begin{array}{l}\text { Participación relativa de los ingresantes de } \\
\text { ingeniera en la matricula de la UNS (\%) }\end{array}$ & 18 & 20 & 22 \\
& 2008 & 2009 & 2010 \\
\cline { 2 - 4 } $\begin{array}{l}\text { Tasa de retención (o reinscripción) } \\
\text { de los alumnos de ingeniería (\%) }\end{array}$ & 81 & 82 & 91 \\
\hline
\end{tabular}

Fuente: elaboración propia en base a datos de la SPU y la UNS

El proyecto presentado y ejecutado por la Universidad Nacional del Sur en la Convocatoria a Proyectos para la Articulación Universidad-Escuela Media realizada por la Secretaria de Políticas Universitarias (SPU) contó con la participación de la Jefatura de Inspección Región XIV Nivel Polimodal Oficial y estuvo destinado a los alumnos del tercer año del polimodal de las ciudades de Bahía Blanca y zona de influencia. Esta convocatoria perseguía establecer acciones conjuntas entre las universidades y las escuelas medias tendientes a promover y facilitar el acceso y permanencia de los estudiantes de las escuelas medias en la educación superior (Ministerio de Educación, Ciencia y Tecnología, 2003). En la concreción de este proyecto se llevaron a cabo diversas jornadas en las que participaron especialistas en temas educativos, docentes y alumnos de nivel medio y superior como así también el dictado de cursos en la UNS y en varias escuelas de la ciudad.

Además en la UNS por Resolución CSU 636/02, se estableció a partir del año 2003, la implementación de un mecanismo de ingreso a las carreras de grado. Cada departamento ${ }^{6}$ define para las carreras bajo su órbita, de forma autónoma, hasta dos asignaturas de nivelación que el alumno deberá aprobar en alguna de las instancias del Programa de Ingreso -Etapa de Nivelación o Ciclo de Articulación. Los contenidos de las mismas se deben ajustar a los alcanzables en el nivel medio y se conciben para cubrir las necesidades cognitivas y de

6 Todos los departamentos de la UNS llevan a cabo este programa a excepción del Departamento de Humanidades, que no solicita ninguna materia de nivelación para los alumnos ingresantes y el de Ciencias de la Salud que implementó un régimen especial 
motivación requeridas para el ingreso a la institución ${ }^{7}$. Cada una de esas materias está vinculada a alguna asignatura del Plan de la Carrera.

Desde 2008, con el objetivo de preparar a los alumnos para la prueba de diagnóstico, comenzaron a dictarse los cursos de Nivelación a distancia de algunas de las asignaturas de nivelación - Matemática, Física, Química y Geografia. Estos cursos están destinados a los preinscriptos en las diferentes carreras y se imparten a través de la plataforma educativa de Continuar.UNS, la cual contiene el material de lectura, las actividades prácticas, y las facilidades de comunicación (foros, e-mail) con los profesores, tutores y compañeros.

Los programas PACENI y PROHUM comprendidos dentro de los Proyectos de apoyo fueron diseñados con el objetivo de mejorar la inserción, promoción y rendimiento académico de los estudiantes del primer año de las carreras de Ciencias Exactas y Naturales, Ciencias Económicas e Informática y de apoyar las actividades que promuevan el mejoramiento de la calidad de la enseñanza en las carreras de la Rama de Ciencias Humanas respectivamente.

En setiembre de 2008, la UNS formalizó la presentación de un proyecto institucional en el marco del PACENI en el que participaron las unidades académicas correspondientes a los Departamentos de Biología, Bioquímica y Farmacia, Ciencias de la Administración, Ciencias e Ingeniería de la Computación, Economía, Física, Matemática, Química, e Ingeniería Química. Con respecto a la implementación del sistema de tutorías prevé incorporar 32 tutores durante un lapso de 36 meses. En lo referente a la mejora de la formación pedagógica de los docentes de primer año prevé mejorar la formación didáctica así como la formación disciplinar de los docentes auxiliares de las asignaturas del primer año de las carreras involucradas en el Programa, mediante el dictado de un curso de capacitación en temas pedagógicos y didácticos por año y de dos cursos de capacitación en temas de desarrollos recientes de las disciplinas. Entre las acciones tendientes a mejorar la intensidad de la formación práctica de los alumnos ingresantes a través de la adquisición de equipamiento, software y bibliografía prevé la adquisición de equipamiento multimedia para su instalación en las aulas, la creación o actualización de Laboratorios de Computación en los Departamentos Académicos involucrados en el Programa, la adquisición de textos para las asignaturas de primer año así como la adquisición de software específicos para materias de primer año de Física y Matemática (UNS, 2008).

7 Una descripción detallada de las características de este programa puede consultarse en Cerioni et al (2008) 
La evaluación de la trayectoria de los alumnos de una cohorte de la UNS realizada mediante la utilización de modelos econométricos muestra al desempeño en la etapa de nivelación como un importante predictor del desempeño académico posterior. Por otro lado, las probabilidades de sortear satisfactoriamente la etapa de nivelación están fuertemente influenciadas por el clima educativo del hogar y por cuestiones relacionadas con el nivel de educación previo tales como el tipo de establecimiento donde cursaron dicho nivel y la concordancia entre modalidad cursada y la carrera elegida.

A partir de esos resultados se evidenciaría la importancia de mejorar la capacitación pedagógica de los docentes que dictan los cursos de nivelación para lograr de este modo incrementar las posibilidades de aprobación de los mismos a la vez que fortalecer el programa de tutorías en esa instancia e intensificar la articulación con el nivel secundario.

\section{III.2. Estrategias tendientes a morigerar las dificultades de orden económico}

Con el objetivo principal de facilitar el acceso, permanencia y egreso de alumnos provenientes de hogares con bajos ingresos, desde la SPU se ofrecen diversos programas de becas: el Programa Nacional de Becas Universitarias (PNBU), el Programa de Becas Bicentenario para Carreras Científicas y Técnicas Programa y el Programa Nacional de Becas para Carreras de grado en Área TICS (PNBTICS).

El PNBU implementado en 1996 está orientado tanto a alumnos como a ingresantes a una carrera de grado de una Universidad Nacional o de un Instituto Universitario Nacional. Es requisito cursar la carrera bajo la modalidad presencial.

El Programa de Becas Bicentenario para Carreras Cientificas y Técnicas que ha lanzado su quinta convocatoria está destinado a alumnos o ingresantes al nivel de educación superior en la rama de las carreras vinculadas a las ciencias aplicadas, ciencias naturales, ciencias exactas y a las ciencias básicas (carreras de grado, tecnicaturas universitarias y no universitarias y profesorados terciarios), consideradas estratégicas para el desarrollo productivo. En el marco de este programa se prevé además realizar una serie de acciones organizadas en tres niveles con la finalidad de lograr una mejor y mayor incidencia del apoyo económico otorgado a los alumnos. Estas acciones tenderían al a) fortalecimiento de las condiciones de base de los alumnos para acceder a la educación superior, b) mejoramiento de las carreras de grado y tecnicaturas prioritarias, c) acompañamiento de los becarios a lo largo de su trayectoria. 
El PNBTICS instituido en 2008, además del objetivo principal de los programas de becas tiene como finalidad fortalecer la formación de recursos humanos en el sector de Tecnologías de la Información y las Comunicaciones.

Los alumnos regulares y los ingresantes de la UNS, además de aplicar a las becas nacionales, cuentan con un menú de becas y subsidios otorgados por la propia universidad. La administración de los mismos está a cargo de la Secretaría General de Bienestar Universitario cuyo objetivo es acompañar a los estudiantes en su paso por la institución ofreciéndoles además de asistencia financiera, atención primaria de la salud, beneficios y descuentos, actividades deportivas y recreativas.

Entre los subsidios se inscriben: un subsidio económico, un subsidio de transporte- que consta de un pasaje ida y vuelta diario, durante el año lectivo para aquellos a estudiantes que vivan a más de 20 cuadras de la UNS, subsidio de comedor- que consiste en un vale por almuerzo y cena de lunes a viernes y almuerzo los días sábado, en el Comedor Universitario-, residencia estudiantil -para estudiantes que provienen de localidades que disten más de $50 \mathrm{~km}$ de Bahía Blanca. Pueden acceder a estos subsidios alumnos de escasos recursos, que cumplan con un rendimiento académico adecuado.

Las becas estímulo que adjudica la UNS tienen como finalidad incentivar a los alumnos en la búsqueda de la excelencia. Se otorga en función de un rendimiento académico sobresaliente, sin tener en cuenta el nivel socio económico del alumno, ya que constituye un antecedente importante para la obtención de futuras becas.

En el corriente año más de 1200 alumnos han recibido subsidios entregados por la UNS, correspondiendo el $57 \%$ de los mismos a subsidios económicos. En tanto que 95 alumnos accedieron a residencias estudiantiles. La cantidad de subsidios distribuidos ha crecido considerablemente en los últimos años a una tasa que supera ampliamente a la incorporación de nuevos alumnos a la institución, asimismo la participación relativa de la partida asignada a becas y subsidios en el presupuesto de la universidad ha registrado variaciones positivas (Cuadro 3, Gráfico 1). 
Cuadro 3. UNS. Subsidios y becas. 2012

\begin{tabular}{lcc}
\hline & $\begin{array}{c}\text { Alumnos } \\
\text { beneficiados }\end{array}$ & $\begin{array}{c}\text { Monto total por tipo } \\
\text { de subsidio }\end{array}$ \\
\hline Subsidios económicos & 699 & $\$ 2.362 .500$ \\
Subsidio transporte & 95 & $\$ 303.964,50$ \\
Subsidio comedor & 306 & $\$ 1.075 .990$ \\
$\begin{array}{l}\text { Residencia estudiantil } \\
\text { Becas de Estimulo al Estudio }\end{array}$ & 215 & $\$ 54.000$ \\
$\begin{array}{l}\text { Total de alumnos beneficiados } \\
\text { Monto de los subsidios } \\
\text { otrogados }\end{array}$ & 1342 & $\$ 3.796 .454,50$ \\
\hline
\end{tabular}

Fuente: elaboración propia en base a datos de la Secretaria de Bienestar Universitario

Gráfico 1. UNS. Subsidios otorgados. Valores absolutos

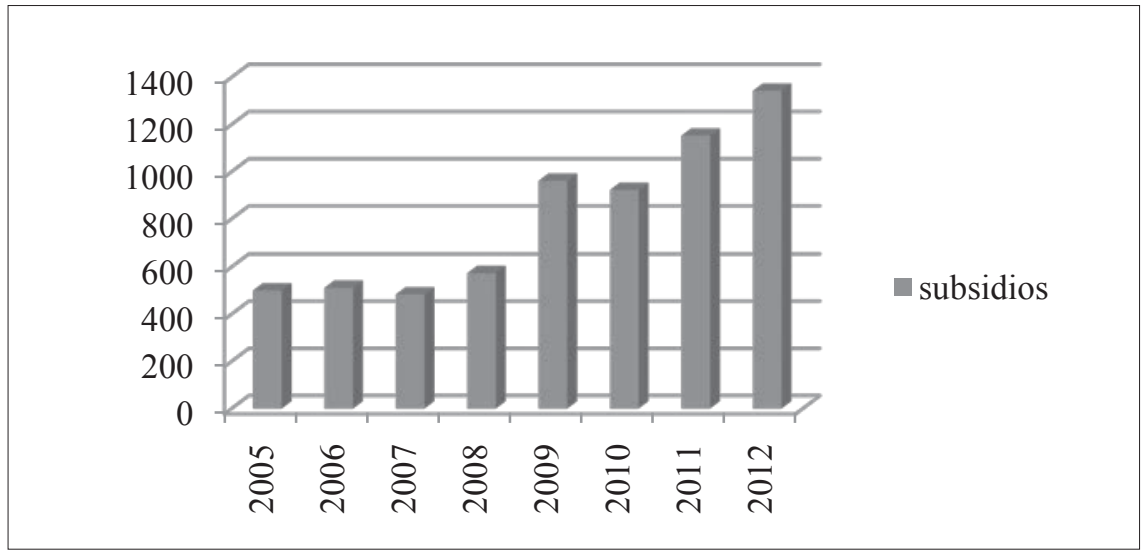

Fuente: elaboración propia en base a datos de la Secretaria de Bienestar Universitario 


\section{A MODO DE REFLEXIÓN}

La problemática del acceso y permanencia en el nivel de educación superior constituye un tema análisis tanto a nivel académico como de las autoridades y una problemática que afecta a la sociedad en su conjunto. Si bien en los últimos años se han implementado diversas medidas para atender las causas que llevan a los estudiantes a abandonar sus estudios, en particular a aquellas relacionadas con dificultades académicas y de orden económico, deberían evaluarse los alcances de estas medidas con la activa participación de la población objetivo para mejorar el diseño de las mismas.

\section{REFERENCIAS BIBLIOGRÁFICAS}

Cerioni, L. \& Morresi, S. (2008). Condicionamientos al ingreso a la educación superior y equidad. Algunos resultados empíricos. Revista Estudios Económicos, XXIV (N.S.) 48, 1-20.

CINDA. (2007). Educación Superior en Iberoamérica Informe 2007. Chile: RIL ${ }^{\circledR}$ Editores.

Di Paola, R., Berges, M. \& Rodríguez, E. (1997). Diferencias de ingreso entre los jefes de familia en la ciudad de Mar del Plata. Un enfoque de la teoría del capital humano, XXXII Anales de la Asociación Argentina de Economía Política. Tomo II

García de Fanelli, A., \& Jacinto C. (2010). Equidad y educación superior en América Latina: el papel de las carreras terciarias y universitarias. Recuperado de http://ries.universia.net

Ministerio de Educación, Ciencia y Tecnología. Secretaría de Políticas Universitarias. (2008). Seminario internacional Diagnóstico y experiencias para la disminución de la deserción estudiantil. Buenos Aires: Autor

Ministerio de Educación, Ciencia y Tecnología. Secretaría de Políticas Universitarias. (2003). Coordinación de Articulación Universitaria. Boletín $\mathrm{N}^{\circ} 1$. Buenos Aires.

Ministerio de Educación. Secretaría de estado de educación y formación profesional. Dirección general de evaluación y cooperación territorial. Instituto de Evaluación. (2011). Panorama de la educación Indicadores de la OCDE 2011, España: Autor.

Ortiz de Guevara E., Morresi, S. Cerioni, L. \& Donnini, N. (2006). Indicadores de Educación superior. La deserción en la UNS. Serie Articulación entre la 
Universidad Nacional del Sur y las escuelas de nivel medio de B. Blanca y su zona de influencia. Bahía Blanca: Ed. Multioffice

Ortiz de Guevara E., Morresi, S. Cerioni, L. \& Donnini, N. (2000). The profile of de university deserter. A compared analysis, Joint Conference IASS- IAOS, Suiza. Recuperado de http//www.iaos2000.admin.ch

Ortiz de Guevara E., Morresi, S. Cerioni, L. \& Donnini, N. (1998). La deserción en la Universidad Nacional del Sur. Bahía Blanca: EdiUNS.

Perlbach de Maradona, I. \& Calderón, M. (1997). Educación y empleo: Un ensayo para Mendoza. XXXII Anales de la Asociación Argentina de Economía Política. Tomo III, 423-443.

Petrecolla, D. \& Botargues, P. (1997). Income distribution and relative economic affluence between populations of income esrners by education in Gran Buenos Aires. XXXII Anales de la Asociación Argentina de Economía Política. Tomo II, 339-360.

Universidad Nacional de Luján (UNLu). (2009). Proyecto Institucional de la UNLu 2009-2012. Recuperado de http://www.unlu.edu.ar

Universidad Nacional del Sur (UNS). (2008). Formulario de presentación del PACENI. Recuperado de www.uns.edu.ar

Rama, C. (2009). La tendencia a la masificación de la cobertura de la educación superior en América Latina. Revista Iberoamericana de Educación, (50), 173-195

Rama, C. (2004). Las nuevas demandadas en educación superior. Recuperado de http://www.claudiorama.name/ensayos.php?pagina $=2$

Siteal. (2010). Resumen estadístico 01. Recuperado de http://www.siteal.iipe-oei.org Secretaría de Políticas Universitarias (SPU) del Ministerio de Educación de la Nación. (2010). Anuario 2010. Buenos Aires: Autor.

(C) 2014 por los autores; licencia otorgada a la Revista Estudios Económicos. Este artículo es de acceso abierto y distribuido bajo los términos y condiciones de la licencia Atribución-No Comercial 3.0 Unported (CC BY-NC 3.0) de Creative Commons. Para ver una copia de esta licencia visite http:// creativecommons.org/licenses/by-nc/3.0/ 